\title{
CORRECTION
}

\section{Correction to: Smacoviridae: a new family of animal-associated single-stranded DNA viruses}

\author{
Arvind Varsani ${ }^{1,2} \cdot$ Mart Krupovic $^{3}[$
}

Published online: 27 July 2018

๑) Springer-Verlag GmbH Austria, part of Springer Nature 2018

\section{Correction to: Archives of Virology (2018) 163:2005-2015 https://doi.org/10.1007/s00705-018-3820-z}

Unfortunately Fig. 6 in Archives of Virology (2018) 163:2005-2015; https://doi.org/10.1007/s00705-018-3820-z is duplicated (Fig. 4). This is corrected in this erratum.

$\triangle$ Mart Krupovic

krupovic@pasteur.fr

1 The Biodesign Center for Fundamental and Applied Microbiomics, School of Life Sciences, Center for Evolution and Medicine, Arizona State University, Tempe, AZ 85287, USA

2 Structural Biology Research Unit, Department of Clinical Laboratory Sciences, University of Cape Town, Observatory 7700, South Africa

3 Unité Biologie Moléculaire du Gène chez les Extrêmophiles, Department of Microbiology, Institut Pasteur, 25 rue du Docteur Roux, 75015 Paris, France 
Fig. 6 Maximum likelihood phylogenetic tree of the $\mathrm{CP}$ amino acid sequences of the smacoviruses inferred using PhyML [6] with the LG+G+I+F substitution model. The phylogenetic tree is midpoint rooted
Genera

Bovismacovirus

Cosmacovirus

Dragsmacovirus

Drosmacovirus

Huchismacovirus

Porprismacovirus

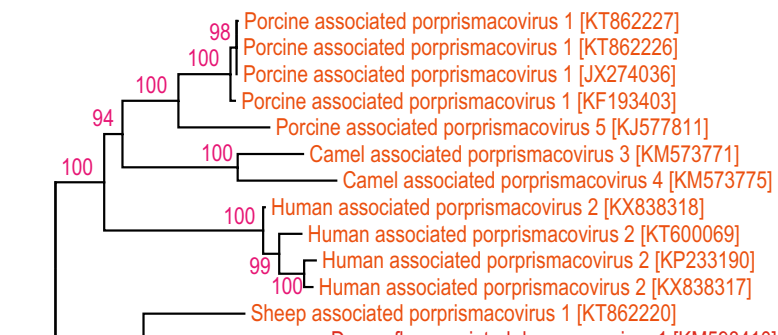

8 Porcine associated porprismacovirus 1 [KT862227]

位

(2)

KM573775

us 2 [KP233190]

Sheep associated porprismacovirus 1 [KT862220] - Dragonfly associated dragsmacovirus 1 [KM598410]

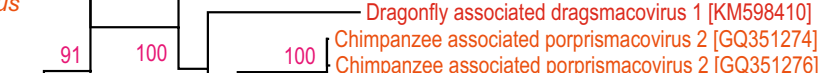

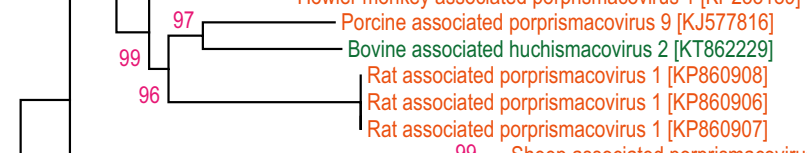

99 Chimpanzes as

99 Porcine associated porprismacovirus 7 [KJ577814]

- Porcine associated porprismacovirus 7 [KJ577815]

Porcine associated porprismacovirus 7 [KJ577812]

991 Porcine associated porprismacovirus 7 [KJ577813]

Howler monkey associated porprismacovirus 1 [KP233189] Porcine associated porprismacovirus 9 [KJ577816]

99 - Sheep associated porprismacovirus 2 [KT862221]

99 Camel associated porprismacovirus 1 [KM573772]

Bovine associated drosmacovirus 1 [KT862224] Bo - Bovine associated huchismacovirus 1 [KT862223]

$79 \longrightarrow$ Camel associated drosmacovirus2 [KM573774]
Sheep associated porprismacos

$100 \quad$ Bovine associated bovismacovirus 2 [KT862222] Bovine associated cosmacovirus 1 [KT862228]

98 Lemur associated porprismacovirus 1 [KP233194]

98 - Turkey associated porprismacovirus 1 [KF880727]

99 Porcine associated porprismacovirus 6 [KJ577819]

100 Porcine associated porprismacovirus 8 [KJ577817]

Porcine associated porprismacovirus 4 [KJ577810]

99 Human associated porprismacovirus 1 [KT600068]

$100 \quad 99$ Chimpanzee associated porprismacovirus 1 [GQ351272]

L Chimpanzee associated porprismacovirus 1 [GQ351275]

Porcine associated porprismacovirus 3 [KC545227]

99 Porcine associated porprismacovirus 3 [KC545229]

100 Porcine associated porprismacovirus 3 [KC545230] Porcine associated porprismacovirus 3 [KC545228]

99 Porcine associated porprismacovirus 2 [KC545226]

Porcine associated porprismacovirus 2 [KJ577818]

99 Human associated huchismacovirus 3 [KP233178]

00 Human associated huchismacovirus 3 [KP233179]

100

- Human associated huchismacovirus 3 [KP264968]

100 Gorilla associated porprismacovirus 1 [KP233191]

Gorilla associated porprismacovirus 1 [KP233192]

Camel associated porprismacovirus 2 [KM573770]

94 - Camel associated porprismacovirus 2 [KM5737

99 Human associated huchismacovirus 2 [KP264967]

86 [Human associated huchismacovius 2 [KP233187]

Human associated huchismacovirus 2 [KP264965]

81100 Human associated huchismacovirus 2 [KP233174]

[ Human associated huchismacovirus 2 [KP233176]

[ Human associated huchismacovirus 2 [KP233177]

Human associated huchismacovirus 2 [KP233175]

Chicken associated huchismacovirus 2 [KY086300]

Chicken associated porprismacovirus 1 [KY086298]

Chicken associated huchismacovirus 1 [KY086301]

- Human associated huchismacovirus 1 [KY086299]

90. Human associated huchismacovirus 1 [KP233186]

96 Human associated huchismacovirus 1 [KP233188]

99 - Human associated huchismacovirus 1 [KP264964]

99 Human associated huchismacovirus 1 [KP233185]

1 d Human associated huchismacovirus 1 [KP233180]

Human associated huchismacovirus 1 [KP233182]

Human associated huchismacovirus 1 [KP264969]

0.5 amino acid substitutions per site

99 Human associated huchismacovirus 1 [KP233193]

- Human associated huchismacovirus 1 [KP233181]

99 Human associated huchismacovirus 1 [KP264966] 